\title{
H1299 Tumor Cell Lysate Vaccine
}

National Cancer Institute

\section{Source}

National Cancer Institute. H1299 Tumor Cell Lysate Vaccine. NCI Thesaurus. Code C114284.

A cell lysate derived from a lung cancer cell line, H1299, with potential immunostimulatory and antineoplastic activities. Upon intramuscular administration, the H1299 tumor cell lysate exposes the immune system to an undefined amount of tumor associated antigens (TAA), particularly cancer testis antigens (CTAs), which may result in the induction of both anti-tumoral cytotoxic T-lymphocytes (CTL) and antibody-dependent responses against TAA-expressing cells, leading to tumor cell lysis. CTAs, such as MAGE, are selectively expressed in a variety of cancers but are not expressed in normal, healthy cells outside the testis. 